\title{
Ion mobility-mass spectrometry and orthogonal gas-phase techniques to study amyloid formation and inhibition
}

\author{
- Amyloids in the gas phase - \\ Waldemar Hoffmann ${ }^{1,2}$, Gert von Helden ${ }^{2}$ and Kevin Pagel ${ }^{1,2}$
}

\begin{abstract}
Amyloidogenic peptide oligomers are responsible for a variety of neurodegenerative disorders such as Alzheimer's and Parkinson's disease. Due to their dynamic, polydisperse, and polymorphic nature, these oligomers are very challenging to characterize using traditional condensed-phase methods. In the last decade, ion mobility - mass spectrometry (IM-MS) and related gas-phase techniques have emerged as a powerful alternative to disentangle the structure and assembly characteristics of amyloid forming systems. This review highlights recent advances in which IM-MS was used to characterize amyloid oligomers and their underlying assembly pathway. In addition, we summarize recent studies in which IM-MS was used to size- and mass-select species for a further spectroscopic investigation and outline the potential of IM-MS as a tool for the screening of amyloid inhibitors.
\end{abstract}

\author{
Addresses \\ ${ }^{1}$ Institute of Chemistry and Biochemistry, Freie Universität Berlin, Takustrasse 3, Berlin 14195, Germany. \\ ${ }^{2}$ Fritz-Haber-Institut der Max-Planck-Gesellschaft, Faradayweg 4-6, Berlin 14195, Germany. \\ Corresponding author: Pagel, Kevin \\ (kevin.pagel@fu-berlin.de)
}

\section{Introduction}

Amyloid formation is a central pathological feature of a variety of neurodegenerative disorders such as Alzheimer's (AD) and Parkinson's disease.[1] In their native state, the involved proteins are usually harmless, soluble and can range from functional, fully folded species to intrinsically disordered structures. Upon environmental changes, such as a change in $\mathrm{pH}$ or interactions with lipid membranes, the proteins undergo a conformational transition and self-assemble into insoluble, $\beta$ sheet rich amyloid fibrils. These fibrils are the major constituents of amyloid plaques that can for example be found in the brain tissue of Alzheimer's patients and were therefore long considered to be the toxic species that cause these diseases. Over the last few years, however, increasing evidence suggested that not the mature fibrils, but rather early, soluble intermediates, that occur during amyloid formation, are the actual toxic species in various neurodegenerative disorders.[2] Due to their polydisperse, polymorphic, and transient nature these intermediates are challenging to characterize via traditional condensed-phase methods like nuclear magnetic resonance (NMR) or circular dichroism (CD) spectroscopy. In most of the cases only ensemble-averaged information is obtained, and as a result, very little is known about the structure of individual oligomers.

Over the last decade, several gas-phase techniques, particularly ion mobility - mass spectrometry (IMMS), emerged as promising tools to separate and analyze individual oligomeric species without affecting the equilibrium of the ensemble.[3] An IM-MS analysis typically starts with the preparation of the sample under conditions where fibril formation occurs. Soluble oligomers are then carefully transferred into the gas phase using soft ionization techniques such as electrospray ionization (ESI). Packets of these ions are further injected into an ion mobility cell that is filled with an inert buffer gas, through which they migrate due to a weak electric field that is applied. During this drift, compact ions undergo fewer collisions with the buffer gas than extended species and therefore traverse the cell faster, i.e. with a shorter drift time. As a result, ions are not only separated according to their mass and charge like in conventional MS, but also based on their rotationally averaged collision-cross section (CCS). This analysis based on size and shape enables the analysis of species, which exhibit an identical $\mathrm{m} / \mathrm{z}$ but differ in their oligomer size or conformation. This review summarizes the recent achievements in which IM-MS and orthogonal techniques were used to obtain detailed structural information of soluble intermediates and to study the mode of inhibition on distinct oligomeric states. 


\section{The Pathway of Amyloid Formation}

Even though the proteins that are involved in amyloid formation differ significantly in their size and sequence, they share similar assembly characteristics.[3] The pathway usually follows a nucleationdependent mechanism, which can be divided into three parts: a lag-, growth- and saturation-phase (Figure 1). Dye molecules such as thioflavin T (ThT) are commonly used to monitor the kinetics of fibril formation in real-time. ThT binds to amyloid fibrils via intercalation, which results in a change in the structure of the dye and an increased fluorescence.[4] It is believed that protein aggregation is initiated by an unfolding/misfolding event of the monomer, forming species that are capable of undergoing oligomerization (lag phase).[3] These oligomers can further exchange subunits or undergo conformational transitions, but once a so-called nucleus is formed, fibril formation is facilitated by an autocatalytic growth (growth phase) up to the presence of mature fibrils (saturation phase). The autocatalytic growth can be further accelerated by fibril fragmentation, providing additional fibril ends for monomer association, whereas end-joining of two fibrils might reduce the rate at which mature fibrils are formed.[5] A detailed understanding of the underlying structures in each phase is crucial for identifying potential targets and the successful development of drugs.

The duration of the lag phase is highly dependent on environmental conditions[6] or the presence of small molecules that can inhibit fibril formation (Figure 1, blue trace).[7] Also the dynamics of the oligomers can lead to different behavior as was for example demonstrated for beta-2 microglobulin (WT) and its H51A alloform.[8] Both proteins form oligomers that are indistinguishable via IM-MS (same CCSs), but the lag phase of the H51A alloform is significantly longer than that of the WT. IMMS and real-time MS using ${ }^{15} \mathrm{~N}$ enriched proteins were performed to study subunit exchange at the end of the individual lag phases. H51A oligomers were shown to undergo no, or a barely measurable exchange, which indicates that these oligomers are kinetically trapped species. WT oligomers, on the other hand, exhibited a rapid subunit exchange, and this behavior accounts for the short lag phase of WT beta-2 microglobulin.[8]

In the presence of pre-formed nuclei that can act as fibrillar seeds, the entire lag phase can be eliminated (Figure 1, red trace).[3] The fibrillar seeds presumably act as a template for monomers to refold and assemble into amyloid fibrils. For example, fibrillar amyloid- $\beta 40$ (A $\beta 40)$ can cross-seed the human islet amyloid polypeptide (hIAPP). The reverse, however, does not occur, i.e. hIAPP seeds are not capable of cross-seeding A 340 monomers. The cross-seeding phenomenon is therefore in many cases sequence dependent. If both peptides are co-incubated another effect termed co-polymerization occurs. Here, mixed A $440 / h I A P P$-oligomers and mixed fibrils are observed. For that reason the crossseeding phenomenon has to be distinguished from co-polymerization.[9]

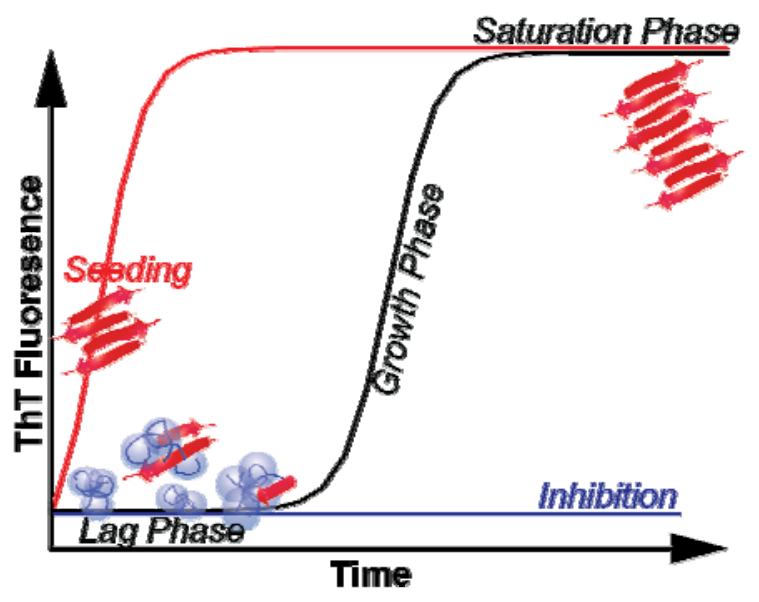

Figure 1: Features of amyloid formation. The typical amyloid formation pathway can be divided into three stages, a lag-, growth- and saturation phase (black trace). During the lag phase, transient and highly interconverting intermediates are found, but no fibrils are present. Once a so-called nucleus is formed, fibril formation is initiated (growth phase), and an autocatalytic growth phase leads to mature fibrils that can be observed at the saturation phase. The addition of nuclei in the form of seeds 
eliminates the lag phase and directly initiates the growth phase (red trace). In the presence of small inhibitors, no fibril formation occurs (blue trace).

\section{Assembly and Architecture of Fibrils}

In principle, all peptides and proteins are able to form amyloids under certain conditions.[6] However, certain amino acids (AAs), exhibit a higher propensity towards fibril formation than others.[10] Recently, it was proposed that the tendency to form amyloid fibrils, microcrystals or unordered aggregates is linked to the propensity of isolated AAs to form large meta clusters.[11] Hydrophobic residues, like isoleucine or phenylalanine, form extended oligomers which are larger in size than expected for an idealized spherical growth of the oligomers.[12] Hydrophilic AAs (serine, asparagine), on the other hand, assemble into more compact oligomers. Peptides, containing multiple hydrophobic AAs (extended AA meta-clusters) are prone to fibril formation, whereas sequences rich in hydrophilic residues (compact AA meta-clusters) are more likely to form microcrystals. This correlation in the assembly characteristics was used to establish a novel approach to score and predict the aggregation propensity of peptides.

Due to extensive research in the last 25 years, the structure of mature fibrils is well understood today. X-Ray diffraction and NMR structures are available for a couple of smaller peptides[13,14] up to fibrils of the full-length $A \beta$ peptide[15,16]. All known structures share a common fibrillar core-structure, often referred to as a "steric-zipper", which is formed by two $\beta$-sheets, each $\beta$-strand stacked in-register.[14] The protruding side-chains of each strand interdigitate like a zipper, forming a dry, tightly selfcomplementing interface. This highly ordered arrangement explains the stability of amyloid fibrils, their self-seeding characteristic and their tendency to form polymorphic structures.

Studying the properties of mature amyloid fibrils using gas-phase techniques is rather challenging due to their enormous size. However, charge-detection mass spectrometry, a method in which an ion's $\mathrm{m} / \mathrm{z}$-ratio and charge are directly measured, was recently used to detect and obtain growth and kinetic characteristics of fibrils as large as $395 \mathrm{MDa}$.[17] Another promising approach to yield dynamic information about mature fibrils is hydrogen-deuterium exchange (HDX), a method in which the solvent accessibility is measured. These experiments revealed that fibrils are not static oligomers, but rather represent a dynamic assembly at the ends of which monomer units dynamically dissociate and associate.[18] This "molecular recycling" takes place on biologically relevant time scales [19] and therefore mature fibrils may serve as a potential source of toxic oligomers [20]. A kinetic-pulsed variation of HDX was also used for the structural characterization of early amyloidogenic intermediates.[21] The data show that unstructured aggregates are present at the beginning of the assembly and evolve into conformations in which specific residues are protected against H/D exchange.

\section{From Monomers to Early Oligomers}

Over the last decade, a series of investigations indicated that not the mature fibrils but rather their intermediates represent the toxic species in the above-mentioned diseases. The toxicity appears to result from interactions with cellular membranes, leading to an impairment of fundamental cellular processes.[6] However, the polydisperse, polymorphic and transient nature of these intermediates makes it difficult to characterize them individually and typically only ensemble-averaged information is obtained. The main advantage of MS-based techniques is the ability to analyze one species in the presence of many others without changing the underlying equilibrium. Thus, IM-MS is a perfect tool for the size and conformer-selective sampling of dynamic structures, either to directly study their shape or to serve as a filter for further structural analysis.

IM-MS was recently used to study the aggregation of the human islet amyloid polypeptide (hIAPP) hormone, which is linked to the etiology of type II diabetes[22], and the non-toxic rat IAPP (rIAPP) isoform, which only differs in six amino acids[23]. The data show that the hIAPP monomer $n / z=1 / 4$ ( $n=$ oligomeric number, $z=$ charge) exists in two major conformations, whereas the rIAPP monomer only forms a compact structure (Figure 2 a).[24] Theoretical calculations suggest a helix-coil conformation for the compact hIAPP monomer whereas an extended $\beta$-hairpin structure was proposed for the extended hIAPP monomer (Figure $2 \mathrm{~b}$ ). Both conformations of hIAPP are highly pH-dependent, 
with the extended $\beta$-hairpin conformation being favored at high $\mathrm{pH}$. CD spectroscopy indicates that under high $\mathrm{pH}$ conditions a faster transition to $\beta$-sheet rich structures occurs, and transmission electron microscopy (TEM) confirmed the formation of fibrils for hIAPP.[25] Therefore, it was suggested that the extended $\beta$-hairpin hIAPP monomer might serve as an amyloidogenic precursor from which the formation of a $\beta$-sheet rich dimer is initiated [26].

a)

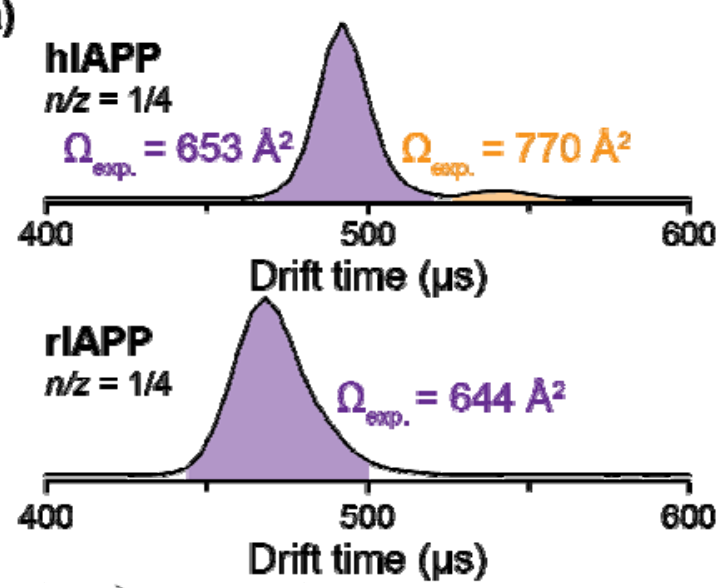

b)

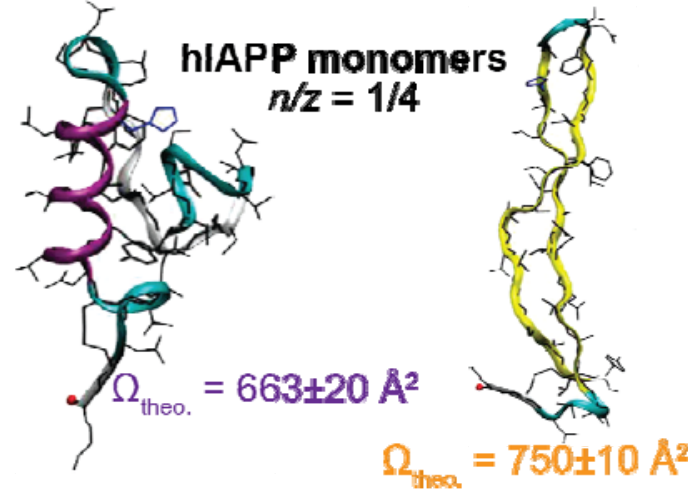

c)
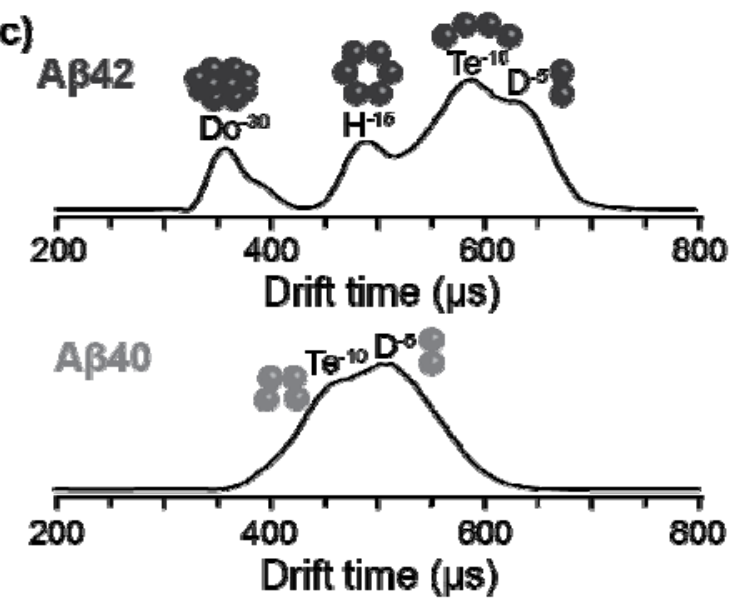

d)

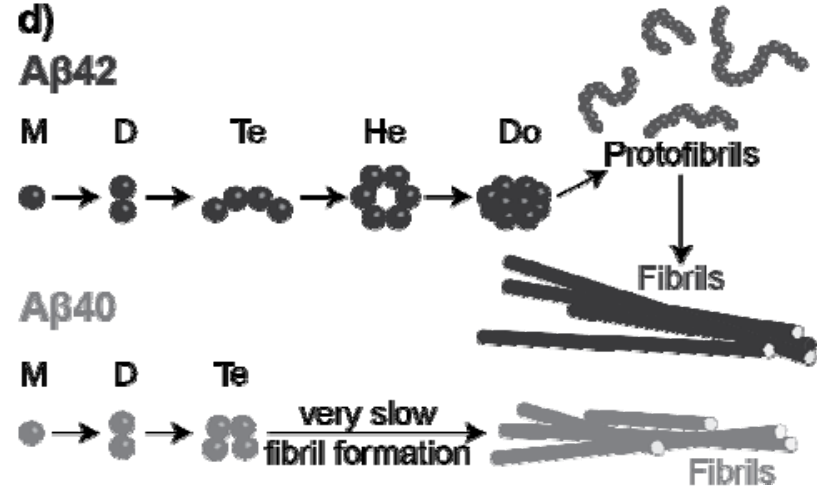

Figure 2: Proposed assembly pathway of IAPP [24] and the AB [27] peptide. (a) The ATD of the quadruply charged hIAPP monomer $(n / z=1 / 4)$ shows two major conformations, whereas for the nonfibril forming rIAPP sequence, only one compact structure is observed. (b) Theoretical calculations have shown that the experimental CCS of the compact hIAPP monomer is in agreement with a helixcoil structure, whereas the extended conformation represents a $\beta$-hairpin structure. Since the $\beta$-hairpin is only present for the fibril forming hIAPP it might be an amyloidogenic precursor. (c) The ATD of the $A \beta 42$ peptide at $\mathrm{m} / \mathrm{z}=1807$ shows four features whereas for the $A \beta 40$ peptide only two features are observed. (d) From this result it was concluded that A 42 forms an "open" tetramer, where a dimer can easily attach to form a hexamer, which then can stack together to form dodecamers. Higher Aß42oligomers are not observed, and the dodecamer is suggested to seed the formation of protofibrils and fibrils. The A 440 peptide, however, forms a "close" tetramer, where no further oligomers can easily attach. As a consequence, A $\beta 40$-fibril formation proceeds very slowly. (Adapted with permission from [24]. Copyright 2009 American Chemical Society. Adapted by permission from Macmillan Publishers Ltd: Nature Chemistry [27], copyright 2009.)

IM-MS is not only suitable providing structural information on the monomer, but it can also be used to study the structure and self-assembly mechanism of higher order oligomers. For example, the peptide NNQQNY was shown to assemble into unordered and compact oligomers up to the octamer, whereas extended and presumably $\beta$-sheet rich structures were observed for the nonamer and larger oligomers.[12] Furthermore, a time-course MS and IM-MS study revealed the dynamic aggregation behavior of a small fragment derived from the human plasma protein transthyretin $\left(\mathrm{TTR}_{105-115}\right)$.[28] The data show that the dimer and tetramer persist over the period of time, whereas other intermediates undergo off-pathway oligomerization or aggregation into fibrils. Thus, the tetramer is suggested to be an on-pathway oligomer, since it represents the smallest unit of a mature fibril, which is assembled by four $\beta$-sheets in a "cross- $\beta$ " conformation. 
Early $A \beta$ oligomers, which are thought to be responsible for the neurodegeneration of $A D$ patients, have also been analyzed using IM-MS.[29] Generally, full-length A 42 aggregates faster and is more cytoxic than the shorter $A \beta 40$ variant [30]. These differences have been explained in detail on basis of IM-MS-derived structural data.[27] A 340 assembles into a compact tetramer (Figure $2 \mathrm{c}, \mathrm{d}$ ), where further peptide monomers cannot easily attach and, as a consequence, no higher oligomers are formed. A $\beta 42$, on the other hand, forms an "open" tetramer, where a dimer can attach to form a hexamer. Two of these hexamers in turn can stack together to form the presumably toxic species in $A D$, the dodecamer [31]. This assembly pathway was first proposed on basis of IM-MS data measured in negative ion mode, but an unambiguous verification based on the isotopic pattern is still missing due to low mass resolution. The assignment is, however, further supported by data obtained from photo-induced cross-linking experiments of unmodified $A \beta$, size exclusion chromatography and dynamic light scattering.[32] Recently, atomic force microscopy was further used to directly monitor the assembly of $A \beta$. The data indicate that dodecamers are on-pathway oligomers, which seed fibrillar aggregates.[33] Furthermore, the neurotoxicity of $A \beta$-oligomers was shown to correlate well with their HDX pattern.[34] $A \beta 40$ and $A \beta 42$ oligomers which persist HDX, i.e. they are at least partly folded and therefore exchange slower, are more cytotoxic than aggregates, which rapidly undergo HDX. In addition, a hydroxyl radical-based, fast photochemical oxidation approach indicates that A $\beta 42$-fibril formation is predominantly driven by the central hydrophobic core (16-27) as well as the C-terminus (28-42), whereas the $\mathrm{N}$-terminus remains almost unstructured [35].

The formation of $A \beta 40$ - and $A \beta 42$-oligomers has also been studied on basis of IM-MS data measured in positive ion mode. At high concentration, the formation of $A \beta 40$ oligomers up to the 13-mer can be observed.[36] A $A 42$ on the other hand, predominantly forms dimers and trimers, which, in the presence of sodium dodecyl sulfate, can further evolve into higher oligomers such as pentamers and hexamers.[37] The difference to the above-described negative ion data may be a result of the different ion polarity and instrument, as well as variations in the sample preparation and further studies are needed to verify the altered assembly behavior.
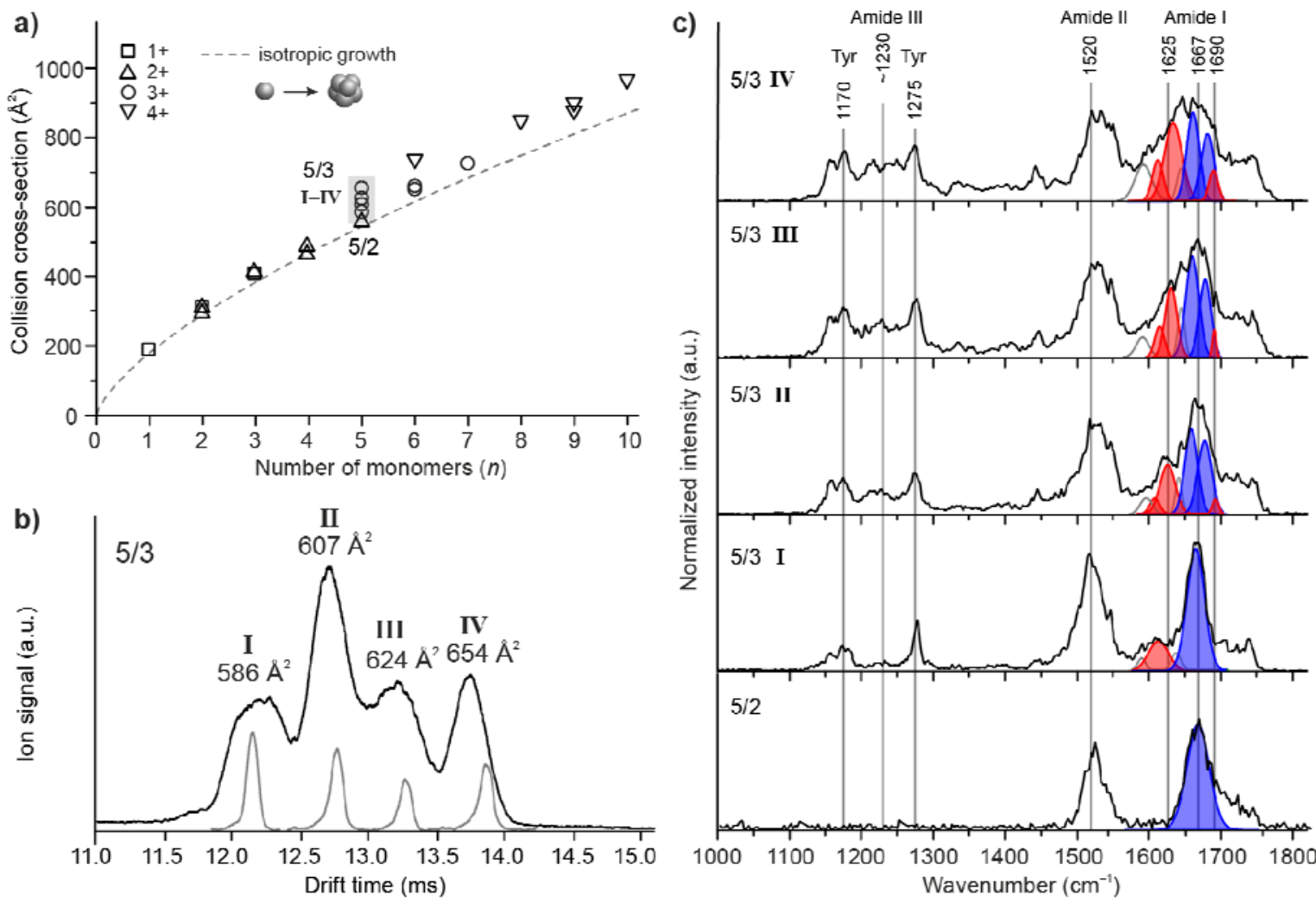

Figure 3: IM-MS data and conformer-selected infrared spectra of the peptide YVEALL.[38] (a) The CCSs are shown as a function of the oligomeric size. The dashed line indicates the CCSs expected for an idealized spherical growth. (b) The arrival time distribution of $n / z=5 / 3$ (black) and selected ions for subsequent IR analysis (gray) are shown. (c) The gas phase IR spectra of the selected YVEALL 
pentamers. The amide I band is deconvoluted with multiple Gaussians, indicating $\beta$-sheet IR signature (red) and turn-like structures (blue). (Adapted by permission from Macmillan Publishers Ltd: Nature Chemistry [38], copyright 2017.)

IM-MS provides valuable data on the ion's overall size and shape, but it does not yield direct information about the underlying internal structure. In order to obtain such data, IM-MS was very recently used as a preselection tool for a further analysis using orthogonal, structure-sensitive gasphase techniques. Action-FRET spectroscopy was coupled to IM-MS to assign the monomeric secondary structure of the $A \beta_{12-18}$ fragment in different charge states. At charge states $3+$ and $4+$ $A \beta_{12-18}$ adopts a predominantly helical conformation, whereas additional protonation $(5+/ 6+)$ leads to a structural transition into a $\beta$-turn.[39] Such charge-dependent conformations are also observed for other proteins in the gas phase.[40,41] Generally, species with a higher charge state form extended structures because Coulomb repulsion and interactions of charged side-chains with backbone carbonyls alter the native fold. For these it is therefore challenging to distinguish if and which of the observed gas phase conformations reflect the "native" species that was present in solution. However, when a protein is carefully transferred into the vacuum using non-denaturating solvents, it has been shown that aspects of the native secondary and tertiary structure can indeed be conserved in the gas phase.[42] In addition, the non-covalent attachment of crown ether molecules to charged side-chains can help to prevent their structurally-disturbing interaction with the protein backbone.[43]

FRET spectroscopy relies on a donor/acceptor system, and therefore a chemical modification is often required, which might alter the native fold. Infrared spectroscopy, on the other hand, can directly probe the secondary structure of peptides and proteins. The position of the $\mathrm{C}=\mathrm{O}$ stretching vibration - often referred to as the amide I band - of $\beta$-sheet rich proteins is usually observed at lower wavenumbers $\left(1620-1640 \mathrm{~cm}^{-1}\right)$ than that of other secondary structure motifs.[44] Recently, the combination of IM-MS and gas phase infrared spectroscopy was used to disentangle the secondary structure of oligomers formed by the peptide VEALYL[12] as well as some of its isomeric variants (YVEALL, VELYAL)[38] (Figure 3). The data show that pentameric oligomers of the peptide YVEALL are highly polymorphic (Figure $3 a$ and $3 b$ ), i.e. they exist in different conformations, which range from compact aggregates with CCSs close to that of an idealized sphere $(n / z=5 / 2)$ to large, extended oligomers for which a $\beta$-sheet rich structure was suggested $(n / z=5 / 3)$. Conformer-specific IR analysis confirmed an increasing $\beta$-sheet content with increasing size (Figure $3 \mathrm{c}$ ), and a more systematic analysis of the investigated amyloid forming hexapeptides revealed a significant amount of $\beta$-sheet for oligomers composed of as little as four to nine subunits. Thus, combining IM-MS to gas phase IR spectroscopy allows one to obtain information not only on the tertiary but also on the secondary structure. These observations further agree with findings from X-ray crystallography experiments, where $\beta$-sheet barrels composed of six strands were observed.[45,46] Moreover, these barrels exhibit theoretically calculated CCSs that are similar to those measured via IM-MS.[47]

\section{Amyloid inhibition}

Drugs often stabilize the native protein structure and are therefore developed using a structure-based design. For (at least partially) unordered peptides and proteins such as those involved in amyloid formation, such a structure-based design is often of limited use and, therefore, low-resolution, highthroughput screening assays are often used as an alternative. IM-MS is capable of high-throughput binding screening with a rate of up to 5000 molecules per day.[7] However, in contrast to other techniques, it also provides information on the stoichiometry and the underlying binding mechanism. It can distinguish between electrostatic interactions, specific (binomial distribution), colloidal and nonspecific binding (Poisson distribution) (Figure 4).[7,48] Competitive interactions can also be studied and, when coupled to fragmentation techniques, complementary information on the stability of oligomers and the binding affinity of the identified potential drugs can be obtained. Therefore, IM-MS is a very valuable tool to screen for amyloid inhibitors.[7]

IM-MS showed that small heat-shock proteins can specifically prevent the aggregation of A 342 and reduce the toxicity in a dose-dependent manner.[49] Molecular tweezers such as CLR01 use electrostatic interactions to bind to lysine 16 and 28 in AB42.[50] This binding affects the structure of the "open" A $A 42$-tetramer, altering it to a more compact conformation similar to the "closed" $A \beta 40$ tetramer. As a consequence, the formation of higher-order oligomers is inhibited.[51] Other IM-MS based studies showed that $\mathrm{Cu}(\mathrm{II})$ ions bind to the histidine 18 residue of hIAPP, which leads to a 
stabilization of off-pathway oligomers and to an inhibition of hIAPP fibril formation.[52-54] A conformerspecific inhibition for hIAPP aggregation was furthermore reported for the molecules silibinin [55] and insulin [56]. Silibinin exclusively binds to the extended $\beta$-hairpin hIAPP monomer (amyloidogenic precursor), and as a result the oligomerization is arrested. In contrast, the helical-rich insulin monomer is proposed to stabilize hIAPP in vivo by interacting with the compact hIAPP monomer. The interaction shifts the hIAPP monomer equilibrium to the compact helix-coil conformation and thereby reduces the abundance of the amyloidogenic precursor.
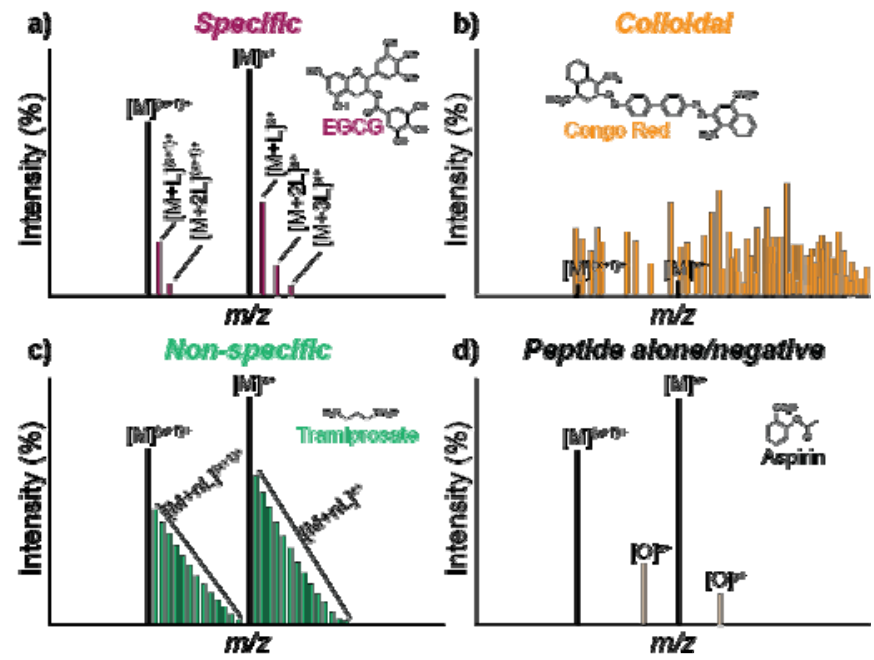

Figure 4: Schematic MS profiling for specific (a), colloidal (b), non-specific (c) and negative (d) interaction between the peptide/protein monomer $(M)$ and potential amyloid inhibitors (L). Example structures for each type of interaction are shown as insets and oligomers are denoted by O. (Adapted by permission from Macmillan Publishers Ltd: Nature Chemistry [7], copyright 2015.)

\section{Conclusion}

In the last decade, the outstanding potential of IM-MS as a tool for the analysis of the structure [24], assembly [27] and dynamics [8] of amyloid oligomers was demonstrated for various examples. Moreover, its impressive potential for amyloid inhibitor screening (with up to 5000 molecules per day) [7] as well as its unique ability to quantify the mode of interaction of inhibitors [48] was shown. Few other techniques are able to access such a wide range of information on such short time scales, and it is therefore likely that the separation power of ESI-IM-MS will contribute significantly to the elucidation of the assembly pathway and the development of novel amyloid inhibitors in the future. IMS on its own is only sensitive to the overall shape of the investigated ions and as such the obtained information content is limited. However, the real strength of the method becomes apparent when it is combined with other orthogonal techniques such as TEM or kinetic assays (e.g. HDX). $[18,34]$ More importantly, also a direct coupling to orthogonal gas-phase techniques can be performed to obtain more detailed structural information. Very recently, IM-MS was for example used as a pre-selection tool to perform gas phase IR [38] or FRET [39] spectroscopy. These experiments led to new insights into the secondary structure of early amyloid intermediates, and it is likely that similar experiments will in the future be applied to study larger amyloid forming systems such as hIAPP and A $\beta$ as well as the binding mode of inhibitors.

\section{Acknowledgements}

This work was supported by the German Academic Exchange Service (DAAD). We thank Prof. Michael T. Bowers for fruitful discussions and continuous support.

\section{References and recommended reading}

Papers of particular interest, published within the period of review, have been highlighted as:

$$
\text { of special interest }
$$


Cole HL et al. Angew. Chem., Int. Ed. 2010, 49:9448-9451: Time-course IM-MS study reveal off- and on-pathway oligomers formed by a small peptide derived from the human plasma protein transthyretin.

Economou NJ et al., J. Am. Chem. Soc. 2016, 138:1772-1775:

A study which demonstrates the ability of AFM to directly follow the assembly of $A \beta 40$ and $A \beta 42$, revealing that the $A \beta 42$-dodecamer is an on-pathway oligomer that seeds fibril formation.

- $\quad$ of outstanding interest Sánchez L et al., J. Am. Chem. Soc. 2011, 133:6505-6508: HDX study which reveals recycli
and Aß42-fibrils. Provides evidence that amyloid recycling occurs on biologically relevant time scales.

HDX study which reveals recycling differences of $A \beta 40-$

Doussineau T. et al., Angew. Chem. Int. Ed. Engl. 2016, 55:2340-2344: Demonstrates how charge detection mass spectrometry coupled to HDX can be used to study the growth and kinetic profile of MDa fibrils.

Seo J. et al., Nat. Chem. 2017, 9:39-44:

Demonstrates the power of IM-MS and gas phase IR spectroscopy to obtain direct secondary structure information of size- and mass-selected amyloid intermediates. Oligomers consisting as little as four to nine subunits were shown to exhibit a significant $\beta$-sheet content.

Young LM et al., Nat. Chem. 2015, 7:73-81: Demonstrates the ability of IM-MS and CID experiments for screening of small inhibitors for amyloid related disorders as well as for studying the mode of action and binding affinities/competitive interactions of inhibitors.

1. Eisenberg D, Jucker M: The amyloid state of proteins in human diseases. Cell 2012, 148:11881203.

2. Kotler SA, Walsh P, Brender JR, Ramamoorthy A: Differences between amyloid-beta aggregation in solution and on the membrane: insights into elucidation of the mechanistic details of Alzheimer's disease. Chem. Soc. Rev. 2014, 43:6692-6700.

3. Woods LA, Radford SE, Ashcroft AE: Advances in ion mobility spectrometry-mass spectrometry reveal key insights into amyloid assembly. Biochim. Biophys. Acta 2013, 1834:1257-1268.

4. Wolfe LS, Calabrese MF, Nath A, Blaho DV, Miranker AD, Xiong Y: Protein-induced photophysical changes to the amyloid indicator dye thioflavin T. Proc. Natl. Acad. Sci. USA 2010, 107:16863-16868.

5. Kalapothakis Jason MD, Morris Ryan J, Szavits-Nossan J, Eden K, Covill S, Tabor S, Gillam J, Barran Perdita E, Allen Rosalind J, MacPhee Cait E: A Kinetic Study of Ovalbumin Fibril Formation: The Importance of Fragmentation and End-Joining. Biophys. J. 2015, 108:2300-2311.

6. Stefani $M$, Dobson $C M$ : Protein aggregation and aggregate toxicity: new insights into protein folding, misfolding diseases and biological evolution. J. Mol. Med. 2003, 81:678-699.

7. Young LM, Saunders JC, Mahood RA, Revill CH, Foster RJ, Tu L-H, Raleigh DP, Radford SE, Ashcroft $A E$ : Screening and classifying small-molecule inhibitors of amyloid formation using ion mobility spectrometry-mass spectrometry. Nat. Chem. 2015, 7:73-81.

8. Leney AC, Pashley CL, Scarff CA, Radford SE, Ashcroft AE: Insights into the role of the beta-2 microglobulin D-strand in amyloid propensity revealed by mass spectrometry. Mol. BioSyst. 2014, 10:412-420.

9. Young LM, Mahood RA, Saunders JC, Tu L-H, Raleigh DP, Radford SE, Ashcroft AE: Insights into the consequences of co-polymerisation in the early stages of IAPP and Abeta peptide assembly from mass spectrometry. Analyst 2015, 140:6990-6999.

10. Caflisch A: Computational models for the prediction of polypeptide aggregation propensity. Curr. Opin. Chem. Biol. 2006, 10:437-444.

11. Do TD, de Almeida NEC, LaPointe NE, Chamas A, Feinstein SC, Bowers MT: Amino Acid Metaclusters: Implications of Growth Trends on Peptide Self-Assembly and Structure. Anal. Chem. 2016, 88:868-876.

12. Bleiholder C, Dupuis NF, Wyttenbach T, Bowers MT: Ion mobility-mass spectrometry reveals a conformational conversion from random assembly to $\beta$-sheet in amyloid fibril formation. Nat. Chem. 2011, 3:172-177. 
13. Nelson R, Sawaya MR, Balbirnie M, Madsen AO, Riekel C, Grothe R, Eisenberg D: Structure of the cross-beta spine of amyloid-like fibrils. Nature 2005, 435:773-778.

14. Sawaya MR, Sambashivan S, Nelson R, Ivanova MI, Sievers SA, Apostol MI, Thompson MJ, Balbirnie M, Wiltzius JJW, McFarlane HT, et al.: Atomic structures of amyloid cross-beta spines reveal varied steric zippers. Nature 2007, 447:453-457.

15. Xiao Y, Ma B, McElheny D, Parthasarathy S, Long F, Hoshi M, Nussinov R, Ishii Y: A[beta](1-42) fibril structure illuminates self-recognition and replication of amyloid in Alzheimer's disease. Nat. Struct. Mol. Biol. 2015, 22:499-505.

16. Wälti MA, Ravotti F, Arai H, Glabe CG, Wall JS, Böckmann A, Güntert P, Meier BH, Riek R: Atomicresolution structure of a disease-relevant $A \beta(1-42)$ amyloid fibril. Proc. Natl. Acad. Sci. USA 2016, 113:E4976-E4984.

17. Doussineau T, Mathevon C, Altamura L, Vendrely C, Dugourd P, Forge V, Antoine R: Mass Determination of Entire Amyloid Fibrils by Using Mass Spectrometry. Angew. Chem., Int. Ed. Engl. 2016, 55:2340-2344.

18. Carulla N, Caddy GL, Hall DR, Zurdo J, Gairi M, Feliz M, Giralt E, Robinson CV, Dobson CM: Molecular recycling within amyloid fibrils. Nature 2005, 436:554-558.

19. Sánchez L, Madurga S, Pukala T, Vilaseca M, López-lglesias C, Robinson CV, Giralt E, Carulla N: $A \beta 40$ and $A \beta 42$ Amyloid Fibrils Exhibit Distinct Molecular Recycling Properties. J. Am. Chem. Soc. 2011, 133:6505-6508.

20. Koffie RM, Meyer-Luehmann M, Hashimoto T, Adams KW, Mielke ML, Garcia-Alloza M, Micheva $\mathrm{KD}$, Smith SJ, Kim ML, Lee VM, et al.: Oligomeric amyloid $\boldsymbol{\beta}$ associates with postsynaptic densities and correlates with excitatory synapse loss near senile plaques. Proc. Natl. Acad. Sci. USA 2009, 106:4012-4017.

21. Carulla N, Zhou M, Giralt E, Robinson CV, Dobson CM: Structure and Intermolecular Dynamics of Aggregates Populated during Amyloid Fibril Formation Studied by Hydrogen/Deuterium Exchange. Acc. Chem. Res. 2010, 43:1072-1079.

22. Pithadia A, Brender JR, Fierke CA, Ramamoorthy A: Inhibition of IAPP Aggregation and Toxicity by Natural Products and Derivatives. J. Diabetes Res. 2016, 2016:2046327.

23. Butler AE, Jang J, Gurlo T, Carty MD, Soeller WC, Butler PC: Diabetes due to a progressive defect in beta-cell mass in rats transgenic for human islet amyloid polypeptide (HIP Rat): a new model for type 2 diabetes. Diabetes 2004, 53:1509-1516.

24. Dupuis NF, Wu C, Shea J-E, Bowers MT: Human Islet Amyloid Polypeptide Monomers Form Ordered $\beta$-hairpins: A Possible Direct Amyloidogenic Precursor. J. Am. Chem. Soc. 2009, 131:18283-18292.

25. Abedini A, Raleigh DP: The Role of His-18 in Amyloid Formation by Human Islet Amyloid Polypeptide. Biochemistry 2005, 44:16284-16291.

26. Dupuis NF, Wu C, Shea J-E, Bowers MT: The Amyloid Formation Mechanism in Human IAPP: Dimers Have $\beta$-Strand Monomer-Monomer Interfaces. J. Am. Chem. Soc. 2011, 133:72407243.

27. Bernstein SL, Dupuis NF, Lazo ND, Wyttenbach T, Condron MM, Bitan G, Teplow DB, Shea J-E, Ruotolo $\mathrm{BT}$, Robinson $\mathrm{CV}$, et al.: Amyloid- $\beta$ protein oligomerization and the importance of tetramers and dodecamers in the aetiology of Alzheimer's disease. Nat. Chem. 2009, 1:326331.

28. Cole HL, Kalapothakis JMD, Bennett G, Barran PE, MacPhee CE: Characterizing Early Aggregates Formed by an Amyloidogenic Peptide by Mass Spectrometry. Angew. Chem., Int. Ed. 2010, 49:9448-9451.

29. Haass C, Selkoe DJ: Soluble protein oligomers in neurodegeneration: lessons from the Alzheimer's amyloid beta-peptide. Nat. Rev. Mol. Cell Biol. 2007, 8:101-112.

30. Selkoe DJ, Hardy J: The amyloid hypothesis of Alzheimer's disease at 25 years. EMBO Molecular Medicine 2016, 8:595-608.

31. Lesné S, Koh MT, Kotilinek L, Kayed R, Glabe CG, Yang A, Gallagher M, Ashe KH: A specific amyloid-beta protein assembly in the brain impairs memory. Nature 2006, 440:352-357. 
32. Bitan G, Kirkitadze MD, Lomakin A, Vollers SS, Benedek GB, Teplow DB: Amyloid $\boldsymbol{\beta}$-protein (A $\boldsymbol{\beta}$ ) assembly: $A \beta 40$ and $A \beta 42$ oligomerize through distinct pathways. Proc. Natl. Acad. Sci. USA 2003, 100:330-335.

33. Economou NJ, Giammona MJ, Do TD, Zheng X, Teplow DB, Buratto SK, Bowers MT: Amyloid $\beta$ Protein Assembly and Alzheimer's Disease: Dodecamers of $A \beta 42$, but Not of $A \beta 40$, Seed Fibril Formation. J. Am. Chem. Soc. 2016, 138:1772-1775.

34. Serra-Vidal B, Pujadas L, Rossi D, Soriano E, Madurga S, Carulla N: Hydrogen/Deuterium Exchange-Protected Oligomers Populated during $A \boldsymbol{\beta}$ Fibril Formation Correlate with Neuronal Cell Death. ACS Chem. Biol. 2014, 9:2678-2685.

35. Li KS, Rempel DL, Gross ML: Conformational-Sensitive Fast Photochemical Oxidation of Proteins and Mass Spectrometry Characterize Amyloid Beta 1-42 Aggregation. J. Am. Chem. Soc. 2016, 138:12090-12098.

36. Kłoniecki M, Jabłonowska A, Poznański J, Langridge J, Hughes C, Campuzano I, Giles K, Dadlez M: Ion Mobility Separation Coupled with MS Detects Two Structural States of Alzheimer's Disease Aß1-40 Peptide Oligomers. J. Mol. Biol. 2011, 407:110-124.

37. Pujol-Pina R, Vilaprinyó-Pascual S, Mazzucato R, Arcella A, Vilaseca M, Orozco M, Carulla N: SDSPAGE analysis of $A \beta$ oligomers is disserving research into Alzheimer's disease: appealing for ESI-IM-MS. Sci. Rep. 2015, 5:14809.

38. Seo J, Hoffmann W, Warnke S, Huang X, Gewinner S, Schöllkopf W, Bowers MT, von Helden G, Pagel K: An infrared spectroscopy approach to follow $\beta$-sheet formation in peptide amyloid assemblies. Nat. Chem. 2017, 9:39-44.

39. Daly S, Kulesza A, Poussigue F, Simon A-L, Choi CM, Knight G, Chirot F, MacAleese L, Antoine R, Dugourd P: Correction: Conformational changes in amyloid-beta (12-28) alloforms studied using action-FRET, IMS and molecular dynamics simulations. Chem. Sci. 2016, 7:1609-1610.

40. Borysik AJ, Kovacs D, Guharoy M, Tompa P: Ensemble Methods Enable a New Definition for the Solution to Gas-Phase Transfer of Intrinsically Disordered Proteins. J. Am. Chem. Soc. 2015, 137:13807-13817.

41. Warnke S, Hoffmann W, Seo J, De Genst E, von Helden G, Pagel K: From Compact to String - The Role of Secondary and Tertiary Structure in Charge-Induced Unzipping of Gas-Phase Proteins. J. Am. Soc. Mass Spectrom. 2016, DOI: 10.1007/s13361-016-1551-5.

42. Seo J, Hoffmann W, Warnke S, Bowers MT, Pagel K, von Helden G: Retention of Native Protein Structures in the Absence of Solvent: A Coupled Ion Mobility and Spectroscopic Study. Angew. Chem. 2016, 55:14173-14176.

43. Warnke S, von Helden G, Pagel K: Protein Structure in the Gas Phase: The Influence of SideChain Microsolvation. J. Am. Chem. Soc. 2013, 135:1177-1180.

44. Barth A: Infrared spectroscopy of proteins. BBA-BIOENERGETICS 2007, 1767:1073-1101.

45. Laganowsky A, Liu C, Sawaya MR, Whitelegge JP, Park J, Zhao M, Pensalfini A, Soriaga AB, Landau $\mathrm{M}$, Teng PK, et al.: Atomic view of a toxic amyloid small oligomer. Science 2012, 335:12281231.

46. Apostol MI, Perry K, Surewicz WK: Crystal Structure of a Human Prion Protein Fragment Reveals a Motif for Oligomer Formation. J. Am. Chem. Soc. 2013, 135:10202-10205.

47. Do TD, LaPointe NE, Nelson R, Krotee P, Hayden EY, Ulrich B, Quan S, Feinstein SC, Teplow DB, Eisenberg $D$, et al.: Amyloid $\beta$-Protein C-Terminal Fragments: Formation of Cylindrins and $\beta$ Barrels. J. Am. Chem. Soc. 2016, 138:549-557.

48. Young LM, Saunders JC, Mahood RA, Revill CH, Foster RJ, Ashcroft AE, Radford SE: ESI-IMS-MS: A method for rapid analysis of protein aggregation and its inhibition by small molecules. Methods 2016, 95:62-69.

49. Hochberg GKA, Ecroyd H, Liu C, Cox D, Cascio D, Sawaya MR, Collier MP, Stroud J, Carver JA, Baldwin AJ, et al.: The structured core domain of $\alpha \mathrm{B}$-crystallin can prevent amyloid fibrillation and associated toxicity. Proc. Natl. Acad. Sci. USA 2014, 111:E1562-E1570.

50. Sinha S, Lopes DHJ, Du Z, Pang ES, Shanmugam A, Lomakin A, Talbiersky P, Tennstaedt A, McDaniel K, Bakshi R, et al.: Lysine-Specific Molecular Tweezers Are Broad-Spectrum 
Inhibitors of Assembly and Toxicity of Amyloid Proteins. J. Am. Chem. Soc. 2011, 133:1695816969.

51. Zheng X, Liu D, Klärner F-G, Schrader T, Bitan G, Bowers MT: Amyloid $\beta$-Protein Assembly: The Effect of Molecular Tweezers CLR01 and CLR03. J. Phys. Chem. B 2015, 119:4831-4841.

52. Riba I, Barran PE, Cooper GJS, Unwin RD: On the structure of the copper-amylin complex. Int. J. Mass Spectrom. 2015, 391:47-53.

53. Li H, Ha E, Donaldson RP, Jeremic AM, Vertes A: Rapid Assessment of Human Amylin Aggregation and Its Inhibition by Copper(II) Ions by Laser Ablation Electrospray Ionization Mass Spectrometry with Ion Mobility Separation. Anal. Chem. 2015, 87:9829-9837.

54. Lee SJC, Choi TS, Lee JW, Lee HJ, Mun D-G, Akashi S, Lee S-W, Lim MH, Kim HI: Structure and assembly mechanisms of toxic human islet amyloid polypeptide oligomers associated with copper. Chem. Sci. 2016, 7:5398-5406.

55. Young LM, Cao P, Raleigh DP, Ashcroft AE, Radford SE: Ion Mobility Spectrometry-Mass Spectrometry Defines the Oligomeric Intermediates in Amylin Amyloid Formation and the Mode of Action of Inhibitors. J. Am. Chem. Soc. 2014, 136:660-670.

56. Susa AC, Wu C, Bernstein SL, Dupuis NF, Wang H, Raleigh DP, Shea J-E, Bowers MT: Defining the Molecular Basis of Amyloid Inhibitors: Human Islet Amyloid Polypeptide-Insulin Interactions. J. Am. Chem. Soc. 2014, 136:12912-12919. 
TOC Graphic

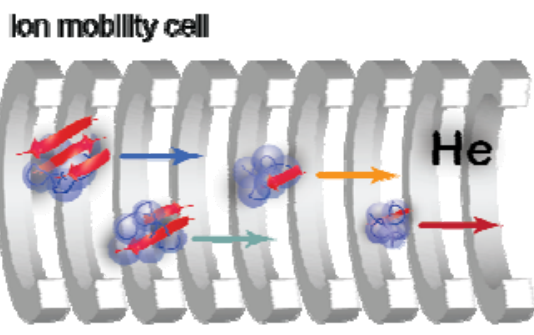

Electrilc fleld $\longrightarrow$

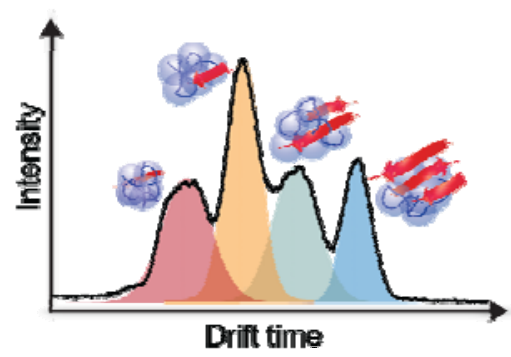

